\title{
Ocorrência de endoparasitas com potencial zoonótico de transmissão em fezes de gatos (Felis catus domesticus Linnaeus, 1758) domiciliados na área urbana e região metropolitana de Castro - Paraná - Brasil
}

The occurrence of endoparasites with zoonotic potential of transmission in feces of cats (Felis catus domesticus Linnaeus, 1758) domiciled in the urban and metropolitan areas of Castro - Paraná-Brazil

\author{
Luiz Carlos Leite'
}

\section{Resumo}

As doenças parasitárias de natureza gastrintestinal destacam-se entre as mais frequentes e importantes afecções envolvendo gatos neonatos, jovens e adultos. Com o objetivo de verificar a ocorrência de endoparasitos, trinta e oito amostras de fezes colhidas de gatos domiciliados na área urbana e região metropolitana da cidade de Castro (PR) foram analisadas, mediante a técnica de Willis. Dezenove amostras $(50,0 \%)$ apresentaram resultado positivo para a presença de ovos e cistos de endoparasitas; houve predomínio de cistos de Cystoisospora spp. em dez (52,63\%). Registrou-se a presença de ovos de Ancylostoma spp. em duas amostras (10,53\%), Toxocara spp. em duas (10,53\%) e cápsulas ovígeras e proglotes de Dipylidium caninum em uma (5,26\%). Foi observada associação entre dois gêneros de parasitas em três amostras: a primeira contaminada com ovos de Toxocara spp. e Ancylostoma spp. (10,53\%), a segunda com cistos de Giardia sp. e Cystoisospora spp. $(5,26 \%)$, enquanto ovos de Toxocara spp. e proglotes e cápsulas ovigeras de Dipylidium caninum tiveram seu achado reportado na terceira amostra (5,26\%). A identificação de diferentes gêneros de endoparasitas comprova o risco potencial de contaminação para outros animais e pessoas e ressalta a importância de um maior controle parasitológico em gatos.

Palavras-chave: gatos; parasitismo; helmintos; protozoa; zoonose.

I Dr.; Médico Veterinário; Professor das Faculdades Integradas do Vale do Iguaçu, UNIGUAÇU; Endereço: Rua Padre Saporti, 7I7, Rio D’Areia, CEP: 84600-000, União da Vitória, Paraná, Brasil; E-mail: Icmouraleite@ gmail.com.br

\begin{tabular}{lllll}
\hline Ambiência Guarapuava (PR) & v.8 n.3 & p. 923 - 930 & Set./Dez. 2012 & ISSN I808 - 025I
\end{tabular}




\section{Abstract}

Parasitic diseases of gastrointestinal kind stand out as the most frequent and important diseases involving newborn, young and adult cats. In order to verify the occurrence of endoparasites, thirty eight feces samples, which were collected from cats residing in the urban and metropolitan areas of the city of Castro-Paraná, were analyzed through the Willis technique. Nineteen samples (50.0\%) tested positive for the presence of eggs and cysts of endoparasites; Cystoisospora spp. Cysts predominated in ten of them (52.63\%). There were eggs of Ancylostoma spp. in two samples (10.53\%), Toxocara sp. in two (10.53\%), and egg capsules and proglottids of Dipylidium caninum in one of the samples (5.26\%). It was also observed the association between two kinds of parasites in two samples. One of them was contaminated with eggs of Toxocara spp./Ancylostoma spp.(10.53\%), and the other one with cysts of Giardia sp./Cystoisospora spp. (5.26\%). Eggs, proglottids and egg capsules of Toxocara spp./Dipilidium caninum were found in one sample (5.26\%). The identification of different kinds of endoparasites is a proof of the potential risk of contamination to other animals and people, emphasizing the importance of greater parasitological control in cats.

Key words: cats; parasites; helminthes; protozoa; zoonosis.

\section{Introdução}

O crescente interesse de pessoas na adoção de gatos abandonados nas ruas vem contribuindo no aumento do número de felídeos domiciliados e semidomiciliados no interior de residências (COELHO et. al., 2010). Paralelamente, infecções gastrointestinais causadas por ampla variedade de espécies de helmintos e protozoários parasitas destes animais, constituem-se em importante fonte de risco à saúde humana, em consequência da contaminação rotineira de crianças e adultos, mediante penetração ativa de larvas infectantes e ingestão de ovos e cistos eliminados em suas fezes (JAFFRY et al., 2009). Gatos podem infectar-se pela transmissão de larvas da fêmea para os filhotes durante a amamentação, ingestão de cistos, ovos e larvas presentes no ambiente e penetração ativa de formas infectantes (FERREIRA et al., 2010).
Animais não domiciliados apresentam maior risco de exposição a infecções parasitárias mediante predação de pequenos roedores, pássaros, artrópodos, ingestão de água contaminada e restos de alimentos encontrados em lixeiras; os domiciliados normalmente habitam locais cujas condições diminuem as possibilidades de contágio. As doenças parasitárias gastrintestinais podem ser diagnosticadas em gatos neonatos, jovens e adultos (TORRICO et al., 2008). Entre as manifestações clínicas mais comuns destacam-se desidratação, diarreia, tosse, prurido anal, enterites provocadas por infecções bacterianas secundárias, vômitos e perda de peso (Silva et al., 2010). Estudos envolvendo parasitismo em gatos no Brasil ainda são escassos, uma vez que a grande maioria concentra-se na avaliação de cães (SERRA et al., 2003).

O cestoda Dipylidium caninum é um helminto parasita de gatos. Normalmente 
nestes animais a infecção não provoca danos de maior gravidade, porém em felinos portadores de grande número de helmintos pode ocorrer irritação da mucosa intestinal e enterite. A saída ativa de proglotes através do ânus pode causar prurido na região perianal. Seres humanos adultos e principalmente crianças podem infectar-se, através da ingestão acidental de pulgas portadoras da larva infectante deste parasita. No Brasil, Marinho e Neves (1979) descreveram dois casos de infecção por $D$. caninum: em uma mulher de 58 anos e uma criança de dois anos de idade. Na cidade de Araguari (MG), Lemos e Oliveira (1985) registraram a parasitose em uma criança que apresentou lesões cutâneas, insônia, irritabilidade, vômitos, perda de apetite e eliminação abundante de proglotes; Molina et al. (2003) no Chile descreveram achado de idêntico parasita em um bebê do sexo feminino com seis meses de idade, diagnosticado pelo encontro de proglotes grávidos nas fezes da criança.

Os nematódeos parasitas Ancylostoma spp. são responsáveis por quadro de diarreia muco-sanguinolenta e anemia hemorrágica em felinos domésticos e silvestres, resultantes da espoliação de sangue. A presença de ulceração na mucosa intestinal está associada à fixação das formas adultas do parasito através de seu aparelho bucal. O quadro é mais severo em gatos jovens. Os animais parasitados emagrecem, podem apresentar desidratação, depressão e diminuição de suas atividades. As larvas destes helmintos causam em seres humanos a afecção denominada "larva migrans cutânea" (LMC) (COELHO et al., 2009), registrada principalmente em países de clima tropical e subtropical, e relacionada ao contato de pessoas adultas e crianças com areia de parques públicos contaminados por larvas de Ancylostoma spp., que penetram através da pele provocando erupção, geralmente de caráter pruriginoso.

Helmintos do gênero Toxocara spp. parasitam o intestino delgado de gatos. Quando a infecção pré-natal é muito acentuada, animais jovens podem vir a óbito em conseqüência da migração das larvas, que provocam lesões hepáticas e pneumonia. Infecções maciças podem resultar em obstruções no sistema digestivo. A ação irritante dos parasitos adultos nas mucosas gástrica e intestinal provoca vômito e diarreia. Podem ocorrer sinais neurológicos associados à ação de toxinas produzidas por vermes vivos ou mortos, e irritação das terminações nervosas intestinais provocadas pela ação de larvas erráticas. As afecções denominadas "larva migrans visceral" (LMV) e "larva migrans ocular" (LMO), caracterizadas por migração errática de larvas infectantes em seres humanos, através do fígado, olhos (PIVETTI-PEZZI, 2009), pulmões, coração, rins e sistema nervoso central podem provocar urticária (MIN-HYE et al., 2010) lesões pulmonares e cardíacas, fadiga, dispnéia e palpitação. Um caso de miocardite e eosinofilia causado por larvas de Toxocara spp. foi reportado por Abe et al. (2002) em um homem com 26 anos de idade.

Os protozoários Cystoisospora spp. e Giardia sp. são responsáveis por surtos em felinos (COELHO et al., 2009) devido à ingestão de água contaminada pelo afluxo de esgotos próximos aos reservatórios. Giardia sp. tem sido descrita como agente responsável por diarreia em gatos, principalmente em filhotes; entretanto o seu verdadeiro potencial ainda não foi esclarecido. Contaminação do meio ambiente e falta de saneamento básico são fatores importantes na ocorrência destas afecções, e o controle é dificultado pela fácil disseminação dos agentes etiológicos. 
Este trabalho teve como objetivo a identificação de ovos, cistos e larvas de endoparasitas em análises de amostras de fezes de gatos domiciliados na área central e região metropolitana do município de Castro (PR), para avaliar a possibilidade da transmissão de parasitas com potencial zoonótico nestes locais.

\section{Material e Métodos}

O estudo foi realizado na cidade de Castro, (Figura 1) situada na região Oeste do estado do Paraná (latitude 24 47’27” Sul e longitude $50^{\circ} 00^{\prime} 43^{\prime \prime}$ Oeste). De acordo com o último censo, (IBGE, 2010) esta localidade possui 16.619 habitantes, com média de 3,4 moradores por domicílio (18). Foram coletadas amostras de fezes de gatos domiciliados, pertencentes a proprietários residentes nas áreas central e metropolitana. Foram avaliados trinta e oito animais, vinte e seis machos e doze fêmeas, com diferentes faixas etárias. $\mathrm{O}$ material foi coletado individualmente, mantido em refrigeração e analisado

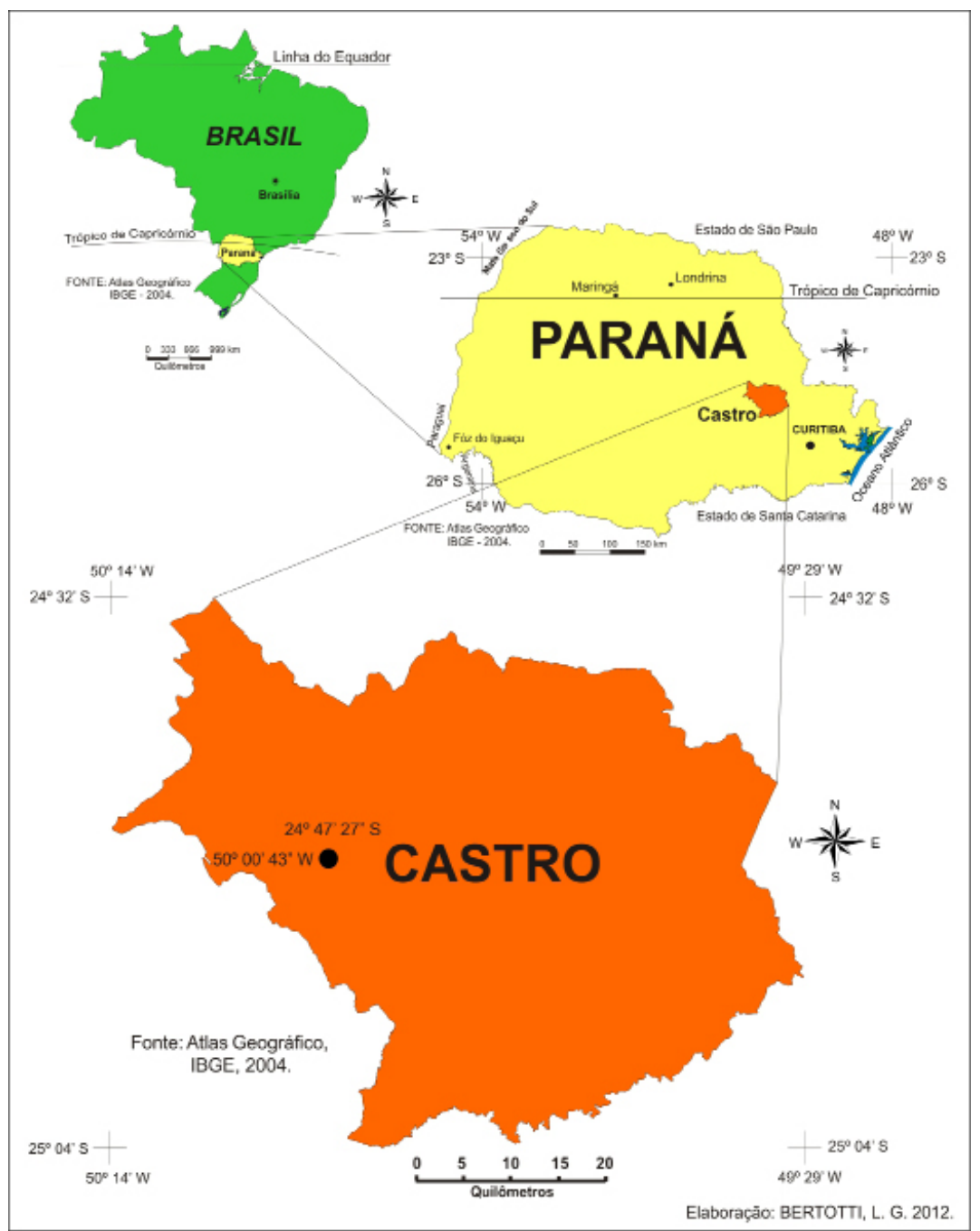

Figura I. Mapa de localização da área de estudo 
mediante exames coproparasitológicos no Laboratório PET-IMAGEM Diagnósticos Veterinários, no máximo em 24 horas após a coleta, pela técnica de flutuação simples em solução saturada de cloreto de sódio (densidade específica de 1,20) descrita por Willis (1921), sendo o resultado considerado positivo quando um ou mais ovos de helmintos, oocistos e formas móveis de protozoários, cápsulas ovígeras e/ou proglotes de Dipylidium caninum fossem identificados conforme suas características morfológicas em exame preliminar ou ao microscópio óptico.

\section{Resultados e Discussão}

De trinta e oito amostras de fezes pesquisadas, dezenove (50,0\%) apresentaram resultado positivo para uma ou mais espécies parasitárias, sendo quinze $(78,96 \%) \mathrm{com}$ uma única parasitose e quatro $(21,04 \%)$ mediante associação de dois gêneros de parasitas. Dezenove amostras (50,0\%) não apresentaram contaminação.

Cistos de Cystoisospora spp. foram identificados em dez amostras analisadas (52,63\%). Foram detectadas duas amostras contaminadas com ovos de Ancylostoma spp. (10,53\%), enquanto ovos de Toxocara spp. foram identificados em duas (10,53\%) e Dipylidium caninum em uma $(5,26 \%)$. Em relação às associações envolvendo dois gêneros de parasitas, ovos de Toxocara spp. e Ancylostoma spp. foram encontrados em duas amostras avaliadas (10,53\%), cistos de Giardia sp. e Cystoisospora spp. em uma $(5,26 \%)$ e ovos de Toxocara spp. e cápsulas ovígeras e proglotes de Dipylidium caninum em uma $(5,26 \%)$ (Tabela 1$)$.

$O$ índice de maior frequência encontrado nesta pesquisa $(52,63 \%)$ foi observado em relação a parasitismo por
Tabela I. Porcentagem de amostras fecais positivas em gatos

\begin{tabular}{lcc}
\hline Parasito & $\begin{array}{c}\text { No de } \\
\text { Amostras }\end{array}$ & $\begin{array}{c}\text { Amostras } \\
\text { Positivas(\%) }\end{array}$ \\
\hline Cystoisospora spp & 10 & 52,63 \\
$\begin{array}{l}\text { Ancylostoma spp } \\
\text { Toxocara spp }\end{array}$ & 2 & 10,53 \\
Dipylidium & 2 & 10,53 \\
caninum & 1 & 5,26 \\
$\begin{array}{l}\text { Toxocara spp / } \\
\text { Ancylostoma spp }\end{array}$ & 2 & 10,53 \\
$\begin{array}{l}\text { Giardia ssp/ } \\
\text { Cystoisospora spp }\end{array}$ & 1 & 5,26 \\
$\begin{array}{l}\text { Toxocara spp / } \\
\text { Dipylidium caninum }\end{array}$ & 1 & 5,26 \\
\hline Total & 19 & 100,00 \\
\hline
\end{tabular}

Cystoisospora spp. Registros envolvendo menores porcentagens foram descritos por Dall'agnol et al.(2010) em Santa MariaRS (17,2\%) e Ragozo et al. (2002), nas cidades de São Paulo e Guarulhos (26,09\%). Igualmente Serra et al. (2003) obtiveram resultados inferiores de contaminação no Rio de Janeiro (43,5\%). Também inferiores ao obtido no presente trabalho foram os percentuais registrados por Jittapalapong et al. (2007) em Bangkok-Tailândia (1,0\%).

A quantidade de amostras que continham ovos de Ancylostoma spp. (10,53\%), foi menor que o resultado encontrado na pesquisa de Coelho et al. $(2010)$ na cidade de Andradina-São Paulo $(98,0 \%)$ e igualmente inferior ao registrado por Dall'Agnol et al. (2010) em Santa Maria-RS (18,1\%). Entretanto, superou os índices registrados por Changizi et al. (2007) no Iran (4,0\%), e os obtidos por Jittapalapong et al. (2007) em Bangkok-Tailândia (9,9\%).

O total de amostras infectadas por ovos de Toxocara spp. neste estudo (10,53\%) foi menor quando comparado aos resultados 
obtidos por Coelho et al.(2010) em AndradinaSão Paulo $(47,3 \%)$ e superior aos resultados obtidos por Dall'Agnol et. al. (2010) em Santa Maria-RS (0,86\%) e por Jittapalapong et al. (2007) em Bangkok-Tailândia (3,5\%). Foi inferior aos reportados por Changizi et al. (2007) e Arbabi e Hooshyar (2009) nas cidades de Bandar-e-Anzali (8,0\%) e Kashan (13\%), no Iran; e menor que os obtidos por Cardillo et al. (2008) em Buenos Aires Argentina (25,0\%).

Foram registrados achados de ovos de Dipylidium caninum em 5,27\% das amostras analisadas, superando os percentuais registrados (0,14\%) em Bangkok-Tailândia por Jittapalapong et al. (2007), porém em menor porcentagem que os reportados por Arbabi e Hooshyar (2009) na cidade de Kashan-Iran (68,1\%). No Brasil, em estudos semelhantes, Dall'Agnol et al. (2010) em Santa Maria-RS descreveram positividade de 1,70\% e Mundim et al. (2004) em UberlândiaMG comprovaram frequência de 14,0\%.

A associação parasitária mais frequente em um mesmo animal envolveu as espécies Ancylostoma spp. e Toxocara spp. $(10,52 \%)$, também consideradas como as mais prevalentes por Ragozo et al. (2002), que registraram positividade de 7,97\% em seu trabalho de pesquisa. Serra et al. (2003) obtiveram índices de 4,61\%, inferiores ao obtido nesta pesquisa.

\section{Conclusão}

O trabalho procurou demonstrar os índices parasitários diagnosticados em amostras de fezes de uma população felina domiciliada das áreas urbana e metropolitana da cidade de Castro (PR). Os resultados encontrados comprovaram infecção por três gêneros de helmintos (Ancylostoma spp., Toxocara spp. e Dipylidium caninum) e dois de protozoários gastrintestinais (Giardia spp. e Cystoisospora spp.). Concluiu-se que as áreas pesquisadas apresentam considerável contaminação por ovos e formas larvais de parasitos, especialmente Ancylostoma spp. e Toxocara spp., importantes gêneros diagnosticados na rotina de inquéritos parasitológicos em todo o Brasil. Estudos envolvendo a frequência do parasitismo em gatos, via de regra, deverão levar em conta o comportamento peculiar destes animais.

\section{Referências}

ABE, K.; SHIMOKAWA, H.; KUBOTA, T.; NAWA, Y.; TAKESHITA, A. Myocarditis associated with visceral larva migrans due to Toxocara canis. Internal Medicine, v.41, n. 9, p.706-708, 2002

ARBABI, M.; HOOSHYAR. Gastrointestinal parasites of stray cats in Kashan, Iran. Tropical Biomedicine, Malaysia, v.26, n.1, p.16-22, 2009.

CARDILLO, N.; ROSA, A.; SOMMERFELT, I. Estudio preliminar sobre los distintos estadios de Toxocara cati en gatos. Parasitologia Latinoamericana, v. 63, n.1-2-3-4, p. 72-75, 2008.

CHANGIZI, E.; MOBEDI, I.; SALAMINI-BEJESTANI, M. R.; REZAEI-DOUST. Gastrointestinal helminthic parasites in stray cats (Felis catus) from North of Iran. Iranian Journal of Parasitology, v.2, n.4, p.25-29, 2007. 
COELHO, N.M.D.; FERNANDES, A.D. S.A.; APOLINÁRIO,J.C.; COELHO,W.M. D.; BRESCIANI, K. D. S. Detecção de helmintos e protozoários de importância em saúde pública em amostras fecais de gatos no município de Andradina, São Paulo. Veterinária e Zootecnia, Botucatu, v.17, n. 1. supl.1, p. 95.2010

COELHO, W. M. D.; AMARANTE, A. F. T.; SOUTELLO, R. V. G; MEIRELES, M. V.; BRESCIANI, K. D. S. Ocorrência de parasitos gastrintestinais em amostras fecais de felinos no município de Andradina. Revista Brasileira de Parasitologia Veterinária, São Paulo, v.18, n.2, p.46-49, 2009.

DALL'AGNOL, L. P.; OTTO, M. A.; SILVA, A. S.; MONTEIRO S. G. Parasitos gastrintestinais em gatos naturalmente infectados no Municipio de Santa Maria no Estado do Rio Grande do Sul, Brasil. Acta Veterinaria Brasilica, v.4, n.3, p. 181-184, 2010.

FERREIRA, D. A.; ALVES, L. C,; FAUSTINO, M. A. G. Ectoparasitos de Felis catus domesticus (Linnaeus, 1758). Revista Biotemas, Florianópolis, v.23, n.4, p.43-50, 2010.

IBGE. Instituto Brasileiro de Geografia e Estatística. Censo 2010. Disponível em: <http:// www.ibge.gov.br/censo2010/index.php>. Acesso em: 12 jan. 2010.

JAFFRY, K. T.; ALI, S.; RASOOL, A.; RAZA, A.; GILL, Z. J. Zoonoses.International Journal of Agriculture \& Biology, v. 11, n. 2, p.217-220, 2009.

JITTAPALONG, S.; INPARNKAEW,T.; PINYOPANUWAT, N.; KENGRADOMKIJ, C.; SANGVARANOND, A.; WONGNAKPHET, S. Gastrointestinal parasites of stray cats in Bangkok Metropolitan areas. Kasetsart, Thailand, v.41, p.69-73, 2007.

LEMOS, C. H.; OLIVEIRA, C. R. Infestação humana pelo Dipylidium caninum. Revista da Sociedade Brasileira de Medicina Tropical, Brasília, v.18, n. 4, p.267-268, 1985.

MARINHO, R. P.; NEVES, D. P. Dipylidium caninum (Dilepididae-Cestoda) - relato de dois casos humanos. Revista do Instituto de Medicina Tropical de São Paulo, São Paulo, v.21, n.5, p.266-268, 1979.

MIN-HYE, K.; JAE-WOO, J.; JAE-WOO, K.; TAE-WHAN, K.; SAE-HOON, K.; SANG-HEON, C.; KYUNG-UP, M.; YOU-YOUNG, K.; YOON-SEOK, C. A Case of Recurrent Toxocariasis Presenting With Urticaria. Allergy Asthma. Immunology Research, v.2, n.4, p.267-270, 2010.

MOLINA, C. P.; OGBURN, J.; ADEGBOYEGA, P. Infection by Dipylidium caninum in an enfant. Archives of Pathology \& Laboratory Medicine, v.157-159. 2003.

MUNDIM, T. C. D.; OLIVEIRA-JÚNIOR, S. D.; RODRIGUES, D. C.; CURY, M. C. Frequência de helmintos em gatos de Uberlândia, Minas Gerais. Arquivos Brasileiros de Medicina Veterinária e Zootecnia, Belo Horizonte, v.56, n.4, p.562-563, 2004. 
PIVETTI-PEZZI, P. Ocular toxocaríasis. International Journal of Medical Sciences, v.6, n.3, p.129-130, 2009

RAGOZO. A. M. A.; MURADIAN, V.; RAMOS e SILVA, J. C.; CARAVIERI, R.; AMAJONER, V. R.; MAGNABOSCO, C.; GENNARI, S. M. Ocorrência de parasitas gastrintestinais em fezes de gatos das cidades de São Paulo e Guarulhos. Brazilian Journal of Veterinary Animal Sciences, v.39, n.5, p. 244-246, 2002.

SERRA, C. M. B.; UCHOA, C. M. A.; COIMBRA, R. A. Exame parasitológico de fezes de gatos (Felis catus domesticus) domiciliados e errantes da Região Metropolitana do Rio de Janeiro, Brasil. Revista da Sociedade Brasileira de Medicina Tropical, Uberaba, v.36, n. 3, p. 331-334, 2003.

SILVA, B. J. de A.; FREIRE, I. M. A.; Da SILVA, W. B.; Do AMARANTE, E. E. V. G. Avaliação das alterações hematológicas nas infecções por helmintos e protozoários em cães (Canis lupus familiaris, Linnaeus, 1758). Neotropical Helminthology, v.41, n. 1, p. 37-48, 2010.

TORRICO, K.J.; SANTOS, K. R.; MARTINS, T.; PAZ E SILVA, F. M.;TAKAHIRA, R.; LOPES, R. S. Ocorrência de parasitas gastrintestinais em cães e gatos na rotina do laboratório de enfermidades parasitárias da FMVZ/UNESP-BOTUCATU, SP. Revista Brasileira de Parasitologia Veterinária, Jaboticabal, v.17, Supl.1, p. 182-183, 2008.

WILLIS, H.H.A simple levitation method for detection of hookworm ova. Medical Journal of Australia, North Sidney, v.8, p. 375-376, 1921. 